\title{
Lapatinib - Member of a New Generation of ErbB-Targeting Drugs
}

\author{
Michael Untch ${ }^{\mathrm{a}}$ Hans-Joachim Lück ${ }^{\mathrm{b}}$ \\ ${ }^{a}$ Frauenklinik/Interdisziplinäres Brustzentrum HELIOS-Klinikum Berlin-Buch, \\ ${ }^{\mathrm{b}}$ Gynäkologisch-onkologische Praxis, Hannover, Germany
}

\section{Key Words}

Trastuzumab resistance - Dual tyrosine kinase inhibitor . Lapatinib · EGF100151 study · Treatment beyond progression

\section{Summary}

Women with advanced or metastatic ErbB2 (HER2)-positive breast cancer have limited therapeutic options once their disease has progressed on trastuzumab-based standard initial chemotherapy regimens. Therefore, there has been a clear need for alternative treatments in this advanced setting. The small molecule lapatinib is a dual receptor tyrosine kinase inhibitor of both ErbB1 and ErbB2. In the pivotal phase III trial, lapatinib combined with capecitabine has demonstrated superior efficacy over capecitabine alone in this group of patients, with a median time to tumor progression of 8.4 months in the combination therapy group versus 4.4 months in the monotherapy arm. This improvement was achieved without an increase in serious toxic effects or symptomatic cardiac events. In addition, with the advent of lapatinib, the empirically adopted practice of continuing trastuzumab beyond progression has to be seen in a different light. This is especially true since an exploratory analysis has suggested that the earlier use of the combination lapatinib plus capecitabine is associated with a benefit in time to progression and overall survival.

\section{Schlüsselwörter \\ Trastuzumab-Resistenz - Dualer Tyrosinkinasehemmer . Lapatinib - EGF100151-Studie - Therapie über den Progress hinaus}

\section{Zusammenfassung}

Für Patientinnen mit fortgeschrittenem oder metastasiertem ErbB2 (HER2)-positiven Mammakarzinom, die unter Therapie mit Trastuzumab-basierten First-line-Standardschemata ein Rezidiv erlitten, waren die Behandlungsoptionen bisher limitiert, so dass für dieses Kollektiv dringend alternative Therapien benötigt wurden. Das «kleine Molekül» Lapatinib hemmt die Tyrosinkinase-Komponenten von ErbB1 and ErbB2. In der Zulassungsstudie erwies sich Lapatinib in Kombination mit Capecitabin der Capecitabin-Monotherapie mit einer medianen Zeit bis zur Tumorprogression von 8,4 Monaten versus 4,4 Monaten deutlich überlegen. Dieser Therapieeffekt konnte ohne höhere Inzidenz schwerwiegender Nebenwirkungen oder symptomatischer kardialer Ereignisse erzielt werden. Darüber hinaus muss mit der Zulassung von Lapatinib die bisher gängige Praxis der Trastuzumab-Therapie über den Progress hinaus überdacht werden. Dies gilt insbesondere vor dem Hintergrund, dass - den Daten einer exploratorischen Analyse zufolge - der frühzeitigere Einsatz von Lapatinib in Kombination mit Capecitabin mit einem Nutzen in Bezug auf die Zeit bis zur Progression und das Gesamtüberleben assoziiert ist.

\begin{tabular}{ll}
\hline KARGER & $\oplus$ 2010 S. Karger GmbH, Freiburg \\
Fax +497614520714 & Accessible online at: \\
Information@Karger.de & www.karger.com/brc \\
www.karger.com &
\end{tabular}




\section{Lapatinib: Mechanism of Action}

To overcome the limitations of treatment with trastuzumab, new agents targeting different signaling pathways were urgently needed. Lapatinib, the first of this new generation of ErbB2 (HER2)-targeting drugs, is an orally active small molecule that is a potent and reversible inhibitor of the ATPbinding site at the tyrosine kinase (TK) domains of the epidermal growth factor receptor (EGFR or ErbB1) and the ErbB2 receptor $[1,2]$. Due to its TK domain inhibition of both ErbB1 and ErbB2, it is classified as a dual TK inhibitor. Lapatinib works intracellularly by reversibly binding to the cytoplasmic ATP-binding site of the kinase and blocking receptor phosphorylation and activation, thereby preventing subsequent downstream signaling events. The ability to specifically inhibit ErbB1 and ErbB2 is unique among the small molecule TK inhibitors, and contrasts with the effects of the licensed TK inhibitors gefitinib and erlotinib which target ErbB1 only [2].

Dual TK inhibitors like lapatinib offer several potential advantages compared with a monoclonal antibody like trastuzumab that targets extracellular ErbB2 only: i) The combined blockade of 2 receptors involved in tumor proliferation may result in a synergistic inhibition of tumor cell growth [3]. ii) The simultaneous inhibition of ErbB1 and ErbB2 is thought to be more effective in preventing the formation of heterodimers involving ErbB1 and ErbB2 [4]. iii) This may result in a more complete inhibition of different signal transduction pathways of the redundant intracellular signaling network [5]. iv) Lapatinib may overcome trastuzumab resistance linked to the overexpression of a truncated ErbB2 (p95). Because lapatinib does not target the extracellular domain of ErbB2, it is able to inhibit p95ErbB2 as has been shown in preclinical models $[6,7]$.

\section{Clinical Phase III Studies of Lapatinib in Combination with Chemotherapy}

Results of a phase III, randomized, open-label study comparing lapatinib in combination with capecitabine or capecitabine alone (EGF100151 study) have first been reported by Geyer et al. [8] and have been updated by Cameron et al. [9]. Women with ErbB2-positive, locally advanced or metastatic breast cancer, that had progressed after treatment with anthracyclines, taxanes, and trastuzumab, were randomly assigned to receive either oral lapatinib $(1,250 \mathrm{mg} /$ day continuously) in combination with oral capecitabine $\left(2,000 \mathrm{mg} / \mathrm{m}^{2} /\right.$ day on days $1-14)$ or capecitabine monotherapy $\left(2,500 \mathrm{mg} / \mathrm{m}^{2} /\right.$ day on days 1-14) every 3 weeks. A total of 155 of 163 women in the combination therapy group (95\%) and 145 of 161 women in the monotherapy group (90\%) received the randomly assigned treatment. The groups were well balanced in terms of patient and disease characteristics. In both groups, more than
$97 \%$ of patients had received prior therapy with anthracyclines, over $98 \%$ were also treated with taxanes, and $97 \%$ had received prior trastuzumab. The median time from discontinuation of trastuzumab to randomization was 5.3 weeks in the lapatinib/capecitabine arm and 6.0 weeks in the monotherapy group. The median duration of previous treatment with trastuzumab was 42 weeks in the combination therapy group and 44 weeks under capecitabine alone. Time to progression (TTP) was defined as the primary endpoint; secondary endpoints included overall survival (OS), progression-free survival (PFS), overall response rate (ORR), and clinical benefit rate $(\mathrm{CBR})$ as well as safety. At the time of the first interim analysis, the primary endpoint TTP met the pre-specified criteria for early reporting on the basis of superiority of the combination arm. Therefore, the Data and Safety Monitoring Committee recommended termination of enrollment. The hazard ratio (HR) for the independently assessed TTP was 0.49 (95\% confidence interval (CI) $0.34-0.71 ; \mathrm{p}<0.001$ ), with 49 events in the combination therapy group and 72 events in the monotherapy group, resulting in a $51 \%$ relative reduction in the risk of disease progression. The median TTP was 8.4 months in the combination group as compared to 4.4 months in the monotherapy group. This improvement was achieved without an increase in serious toxic effects or symptomatic cardiac events. Additional retrospective analyses of trial data showed that the incidence of central nervous system (CNS) or brain metastases as first site of metastasis was lower in patients in the combination treatment arm. Only 4 women receiving the combination therapy experienced CNS relapse versus 11 of those on capecitabine alone ( $p=0.10$ by Fisher's exact test). As concluded by the authors, the results of the EGF100151 study provide support for the use of lapatinib and capecitabine in women with progression of ErbB2-positive breast cancer after treatment with trastuzumab.

These data of the pivotal study have led to he approval of lapatinib (Tyverb ${ }^{\circledR}$, GlaxoSmithKline GmbH und Co. KG, Munich, Germany) in combination with capecitabine for patients with advanced or metastatic ErbB2-positive breast cancer, who have received prior therapy including an anthracycline, a taxane, and trastuzumab in the metastatic setting in 2007 or 2008 in the US and Europe. The recommended dosage of lapatinib is $1,250 \mathrm{mg}$ ( 5 tablets $250 \mathrm{mg}$ each) given orally once daily on days 1-21 continuously in combination with capecitabine $2,000 \mathrm{mg} / \mathrm{m}^{2} /$ day (administered orally in 2 doses approximately $12 \mathrm{~h}$ apart) on days 1-14 in a repeating 21-day cycle. Lapatinib should be taken at least $1 \mathrm{~h}$ before or $1 \mathrm{~h}$ after a meal. The dose should be taken once daily; dividing the daily dose is not recommended. Lapatinib peak plasma levels occur 3-6 $\mathrm{h}$ after oral administration. The effective plasma half-life is approximately $24 \mathrm{~h}$ [10].

The updated analysis of the EGF100151 study continued to show a significant benefit for the combination lapatinib/ capecitabine, consistent with the reported interim analysis. With 184 TTP events identified by independent review ( 82 in 


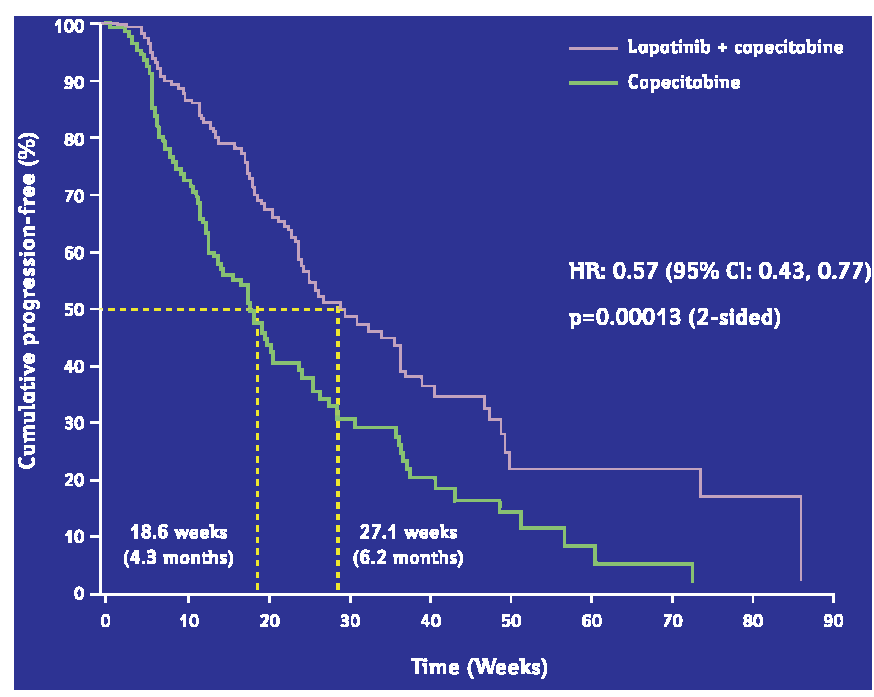

Fig. 1. Update EGF100151 study: Kaplan-Meier estimates of time to progression (intention-to-treat (ITT) population), adapted from [9].

the combination therapy group and 102 in the monotherapy group), there was a significant improvement in median TTP from 4.3 to 6.2 months (fig. 1) [9]. OS was similar (HR 0.78, $95 \%$ CI $0.55-1.12 ; \mathrm{p}=0.177$ ) and fewer cases with CNS involvement at first progression were seen (4 vs. 13).

Di Leo et al. [11] initiated a phase III trial evaluating the efficacy of lapatinib $1,500 \mathrm{mg} /$ day in combination with paclitaxel $\left(175 \mathrm{mg} / \mathrm{m}^{2}\right.$, every 3 weeks) or placebo as first-line therapy in 579 women with metastatic breast cancer with either unknown or negative ErbB2 status (EGF 30001). A preplanned retrospective evaluation of ErbB2 status was performed using fluorescence in situ hybridization (FISH) and immunohistochemistry (IHC). The primary endpoint of the study was TTP; secondary endpoints included objective response rate (ORR), CBR, event-free survival (EFS), and OS. In the intent-to-treat population $(\mathrm{n}=579)$, there were no significant differences in TTP, EFS, or OS between treatment arms, although differences in ORR and CBR were noted. In 86 patients with ErbB2-positive tumors (15\% of the entire trial population), treatment with paclitaxel plus lapatinib resulted in statistically significant improved clinical outcomes. The median TTP was significantly longer in lapatinib-treated patients versus patients receiving placebo (36.4 vs. 25.1 weeks) (fig. 2), with an unadjusted HR of 0.53 (95\% CI 0.31-0.89; $\mathrm{p}=0.005$ ), resulting in a $47 \%$ relative lower risk of progression for patients in the paclitaxel-lapatinib arm versus the paclitaxelplacebo arm. Median EFS was also significantly longer in the paclitaxel-lapatinib arm (35.1 vs. 21.9 weeks; $\mathrm{HR}=0.52 ; 95 \%$ CI $0.31-0.86 ; \mathrm{p}=0.004)$. In addition, ORR (63.3 vs. $37.8 \%$; $\mathrm{p}=0.023)$ and CBR (69.4 vs. $40.5 \% ; \mathrm{p}=0.011)$ were significantly higher for patients treated with paclitaxel-lapatinib compared to paclitaxel-placebo. Median OS was longer in the paclitaxel-lapatinib arm compared to the paclitaxel-placebo arm; however, this difference was not statistically significant.

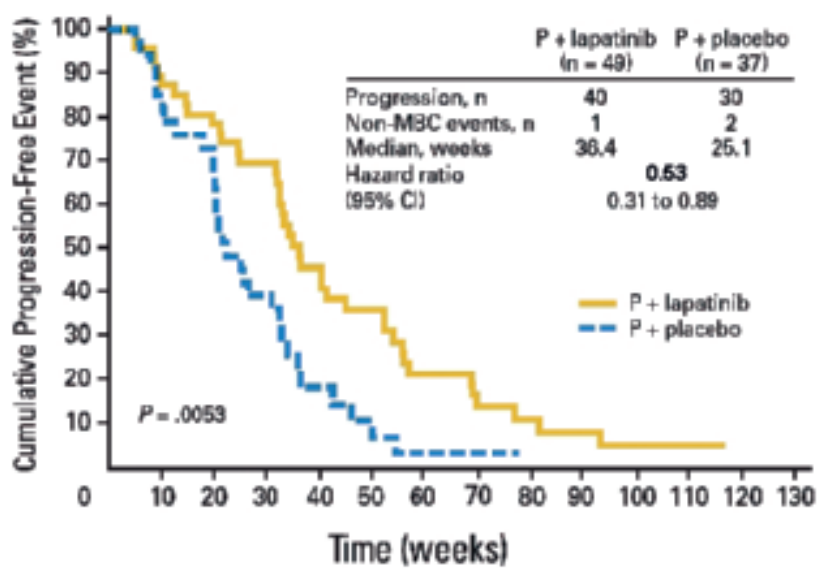

Fig. 2. EGF30001 study: Kaplan-Meier estimates for time to progression in the ErbB2-positive ITT population. The hazard ratio refers to the comparison of paclitaxel $(\mathrm{P})$ plus lapatinib versus $\mathrm{P}$ plus placebo, adapted from [11].

Survival data in the ErbB2-positive population were not mature because only $43 \%$ of events had occurred at the time of data lock. No statistically significant differences between treatment groups were observed for any endpoint in ErbB2negative patients.

\section{Treatment beyond Progression with Trastuzumab or Switch to Lapatinib?}

Since most women with advanced ErbB2-positive breast cancer, who responded to trastuzumab in first-line therapy, will experience progressive disease, the question of the most appropriate second-line treatment has become essential and critical in daily practice. Before the availability of lapatinib, trastuzumab was often empirically continued beyond disease progression, an approach that was deviating from the general oncologic principle of changing treatment at disease progression, and was based only on various retrospective analyses. Attempts in the US and Europe to investigate trastuzumab beyond progression in prospective randomized trials had failed due to poor accrual [12-14].

The introduction of second-generation ErbB2-targeted agents like lapatinib raised a new question: should patients with objective evidence of disease progression be switched to lapatinib/capecitabine or should trastuzumab be continued? More recently, von Minckwitz et al. [12] reported data from the first prospective randomized trial evaluating the benefit of continuing trastuzumab in addition to chemotherapy in ErbB2-positive patients with advanced breast cancer, who had experienced progression while receiving first-line trastuzumab-based chemotherapy. The German Breast Group (GBG) 26/Breast International Group (BIG) 03-05 trial included 156 women randomly assigned to capecitabine alone 


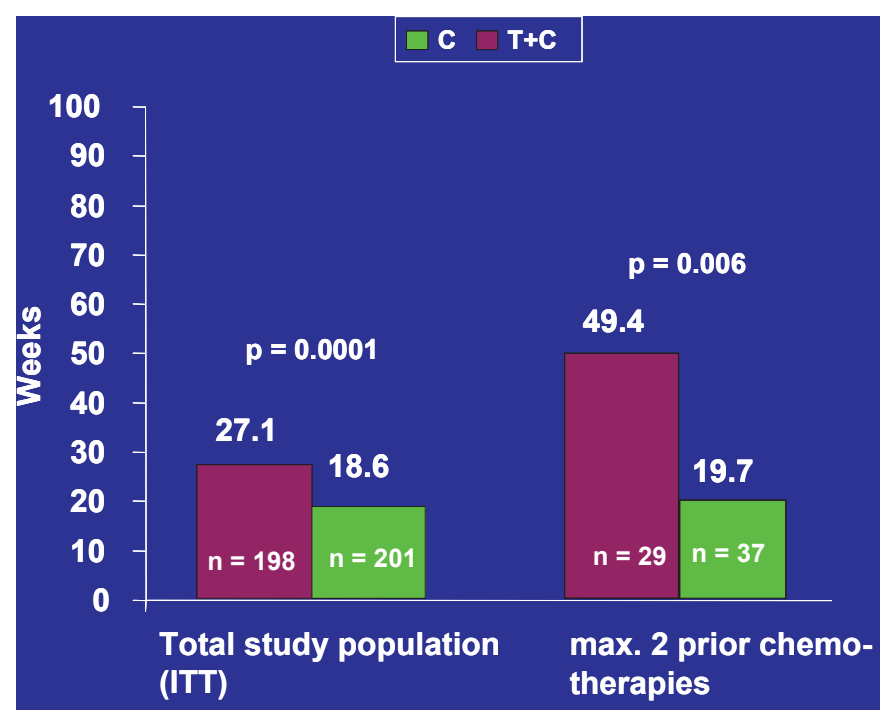

Fig. 3. Exploratory subset analyses of the EGF100151 study: time to progression, adapted from [15].

$\left(2,500 \mathrm{mg} / \mathrm{m}^{2}\right.$ on days 1 through $\left.14 ; \mathrm{n}=78\right)$ or capecitabine plus trastuzumab $(6 \mathrm{mg} / \mathrm{kg} ; \mathrm{n}=78)$ in 3 -week cycles. Previous first-line treatment for metastatic disease consisted of a taxane-plus-trastuzumab combination in 111 patients, trastuzumab alone, or with a non-taxane-containing chemotherapy in 42 patients, and taxane- and trastuzumab-containing adjuvant therapy in 3 patients. The median durations of previous trastuzumab treatment were 45 weeks (range 7-235 weeks) in the capecitabine group and 44 weeks (range 10-284 weeks) in the capecitabine-plus-trastuzumab group. The primary endpoint was TTP. During a median follow-up of 15.6 months, 65 events occurred in the capecitabine group, and 62 events in the combination arm. The median TTP as the primary endpoint of the intent-to-treat analysis was 5.6 months in the capecitabine and 8.2 months in the combination group with an unadjusted HR of 0.69 (95\% CI 0.48-0.97; two-sided log-rank $\mathrm{p}=0.0338$ ). Continuation of trastuzumab beyond progression was not associated with a significant improved OS (capecitabine: 20.4 months $\{95 \%$ CI 17.8-24.7\}, combination group: 25.5 months $\{95 \%$ CI $19.0-30.7\} ; \mathrm{p}=0.257)$. Comparing these results to those of the lapatinib pivotal EGF100151 study has its limitations, as the authors of the GBG26/BIG3-05 study observed. First, the lapatinib trial had a larger sample size with independently assessed response rates and TTP. Secondly, in the EGF100151 study, lapatinib/capecitabine was administered to patients who had received multiple prior regimens, whereas in the GBG26/BIG3-05 trial, the vast majority of the women started capecitabine as second-line chemotherapy for metastatic disease. The higher rate of prior therapies in the EGF100151 study explains the shorter TTP in the capecitabine-alone group (4.4 months) compared with the TTP in the GBG26/BIG3-05 study (5.6 months).

To evaluate the clinically relevant question whether the benefit of lapatinib/capecitabine is maintained or improved

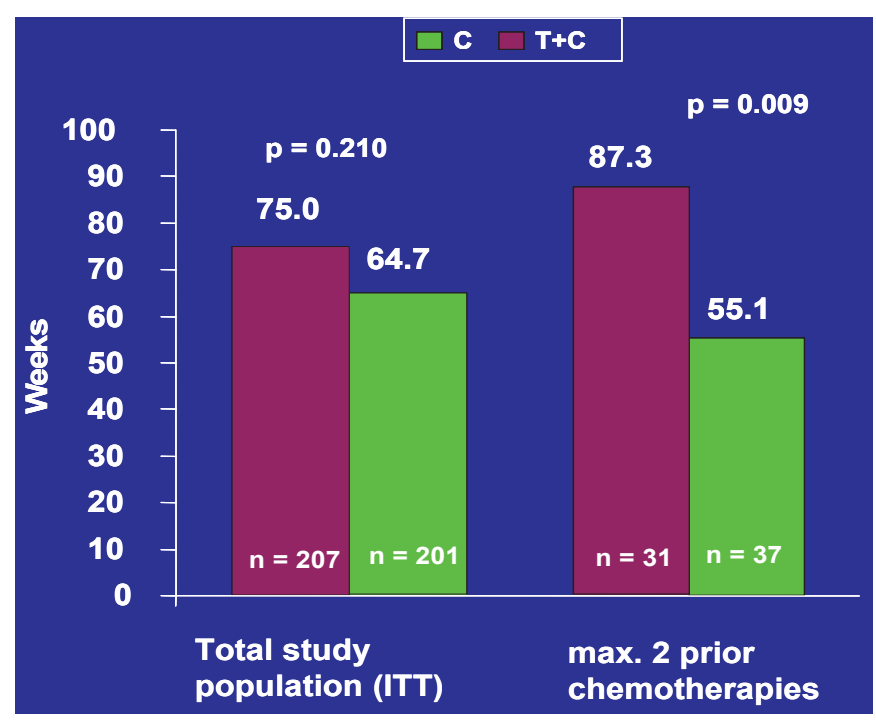

Fig. 4. Exploratory subset analyses of the EGF100151 study: Overall survival, adapted from [15].

by administering the combination therapy in patients with fewer prior regimens, Crown et al. [15] conducted an exploratory subset analyses of the EGF100151 study in patients with fewer prior regimens. A subgroup analysis was performed for patients treated with $<3$ prior regimens, where a regimen was defined as any regimen in any setting (neoadjuvant, adjuvant, or metastatic). These patients with $<3$ prior chemotherapy regimens not only had a significant increase in TTP (19.7 weeks for capecitabine alone vs. 49.4 weeks for lapatinib/ capecitabine, $\mathrm{p}=0.006$ ) (fig. 3) but also, in contrast to the GBG26/BIG3-05 trial, a significant prolongation of OS (55.1 weeks for capecitabine alone vs. 87.3 weeks for lapatinib/ capecitabine; $p=0.009$ ) (fig. 4). Although these data were derived from exploratory analyses, they suggest that the earlier use of the combination lapatinib/capecitabine is associated with a greater benefit concerning both TTP and OS.

Besides the proven efficacy of the dual TK inhibitor after trastuzumab progression, there are several arguments supporting the switch to lapatinib instead of treatment beyond progression with trastuzumab. Unlike trastuzumab, lapatinib offers the convenience of oral administration and has the potential to cross the blood-brain barrier with some evidence of efficacy in treating brain metastases which develop in up to one third of patients with ErbB2-positive metastatic breast cancer [13]. Furthermore, several potential mechanisms of trastuzumab resistance were identified, and there is a growing body of evidence suggesting that lapatinib may be able to overcome this resistance due to its different mechanism of action, targeting against both ErbB1 and ErbB2, and acting intracellularly. For instance, cells expressing the truncated receptor p95ErbB2 were resistant to trastuzumab but remained sensitive to the antiproliferative effects of lapatinib, both in vitro and in vivo $[16,17]$. In addition, loss of PTEN did not affect induction of tumor cell apoptosis by lapatinib in different 
cell lines, suggesting that lapatinib seems to exert its antitumor activity in ErbB2-overexpressing breast cancers in a PTEN-independent manner [18].

Finally, the lapatinib/capecitabine combination is an FDAand EMEA-approved regimen, whereas the combination of trastuzumab with capecitabine has not been approved formally by regulatory authorities.

\section{Disclosure Statement}

The authors were speakers and contributors to the meeting 'ErbB2 (HER2)-positives Mammakarzinom; 2. Münchner Brustkrebs-Symposium Update 2009', 23/24 October 2009 in Munich, sponsored by GlaxoSmithKline.

\section{References}

1 Moy B, Goss P: Lapatinib: current status and future directions in breast cancer. Oncologist 2006;11:1047-1057.

2 Johnston S, Leary A: Lapatinib: a novel EGFR/ HER2 tyrosine kinase inhibitor for cancer. Drugs Today 2006;42:441-453.

3 Burris HA 3rd: Dual kinase inhibition in the treatment of breast cancer. Initial experience with the EGFR/ErbB-2 inhibitor lapatinib. Oncologist 2004; 9(suppl 3):10-15.

4 Rusnak DW, Affleck K, Cockerill SG, et al.: The characterization of novel, dual ErbB-2/EGFR, tyrosine kinase inhibitors: potential therapy for cancer. Cancer Res 2001;61:7196-7203.

5 Nelson MH, Dolder CR: Lapatinib: a novel dual tyrosine kinase inhibitor with activity in solid tumors. Ann Pharmacother 2006;40:261-269.

6 Xia W, Liu LH, Ho P, Spector NL: Truncated ErbB2 receptor (p95ErbB2) is regulated by heregulin through heterodimer formation with ErbB3 yet remains sensitive to the dual EGFR/ErbB2 kinase inhibitor GW572016. Oncogene 2004;23:646-653.

7 Anido J, Scaltriti M, Bech Serra JJ, et al.: Biosynthesis of tumorigenic HER2 C-terminal fragments by alternative initiation of translation. EMBO J 2006;25:3234-3244.
8 Geyer CE, Forster J, Lindquist D, et al.: Lapatinib plus capecitabine for HER-2-positive advanced breast cancer. N Engl J Med 2006;355:2733-2743.

9 Cameron D, Casey M, Press M, et al.: A phase III randomized comparison of lapatinib plus capecitabine versus capecitabine alone in women with advanced breast cancer that has progressed on trastuzumab: updated efficacy and biomarker analyses. Breast Cancer Res Treat 2008;112:533-534.

10 Paul B, Trovato JA, Thompson J: Lapatinib: a dual tyrosine kinase inhibitor for metastatic breast cancer. Am J Health Syst Pharm 2008;65:1703-1710.

11 Di Leo A, Gomez HL, Aziz Z, et al.: Phase III, double-blind, randomized study comparing lapatinib plus paclitaxel with placebo plus paclitaxel as first-line treatment for metastatic breast cancer. J Clin Oncol 2008:26:5544-5552.

12 Von Minckwitz G, du Bois A, Schmidt M, et al.: Trastuzumab beyond progression in human epidermal growth factor receptor 2-positive advanced breast cancer: a German Breast Group 26/Breast International Group 03-05 study. J Clin Oncol 2009;27:1999-2006.

13 Jahanzeb M: Continuing trastuzumab beyond progression. J Clin Oncol 2009;27:1935-1937.
14 Valabrega G, Aglietta M: Trastuzumab beyond disease progression: case closed? J Clin Oncol 2009;27:e121-e122.

15 Crown J, Casey MA, Cameron D, Newstat B, Stein SH: Lapatinib (L) plus capecitabine (C) in HER2+ metastatic breast cancer (MBC): exploratory analyses by prior therapy. Eur J Cancer 2009; 7(suppl):abstr P-5082.

16 Scaltriti M, Rojo F, Ocaña A, et al.: Expression of p95HER2, a truncated form of the HER2 receptor, and response to anti-HER2 therapies in breast cancer. J Natl Cancer Inst 2007;99:628-638.

17 Xia W, Liu LH, Ho P, Spector NL: Truncated ErbB2 receptor (p95ErbB2) is regulated by heregulin through heterodimer formation with ErbB3 yet remains sensitive to the dual EGFR/ErbB2 kinase inhibitor GW572016. Oncogene 2004;23: 646-653.

18 Xia W, Husain I, Liu L, et al.: Lapatinib antitumor activity is not dependent upon phosphatase and tensin homologue deleted on chromosome 10 in ErbB2-overexpressing breast cancers. Cancer Res 2007;67:1170-1175. 